\title{
Fuzzy Sub-vector Spaces and Sub-bivector Spaces under $t$-Norms
}

\author{
Rasul Rasuli \\ Department of Mathematics, Payame Noor University(PNU), Tehran, Iran. \\ rasulirasul@yahoo.com
}

\begin{abstract}
In this paper we introduce and investigate some properties of fuzzy sub-vector spaces and fuzzy sub-bivector spaces by using $t$-norms and consider some of their properties and structured characteristics. Also we define some concepts as intersection, sum and linear transformations for fuzzy sub-vector spaces by using $t$-norms and obtain some results and we establish some of their properties about them and give several examples to illustrate the obtained results.
\end{abstract}

Keywords: Vector spaces, bivector spaces, Linear algebra, Linear transformations, fuzzy set theory, norms, fuzzy sub-vector spaces, fuzzy sub-bivector spaces.

2010 MSC No:15A03, 97H60, 15A04, 03E72, 03B45.

\section{Introduction}

Vector spaces stem from affine geometry via the introduction of coordinates in the plane or three-dimensional space. Around 1636, Descartes and Fermat founded analytic geometry by equating solutions to an equation of two variables with points on a plane curve. In 1804, to achieve geometric solutions without using coordinates, Bolzano introduced certain operations on points, lines and planes, which are predecessors of vectors. His work was then used in the conception of barycentric coordinates by Mobius in 1827. In $1828 \mathrm{C}$. V. Mourey suggested the existence of an algebra surpassing not only ordinary algebra but also two-dimensional algebra created by him searching a geometrical interpretation of complex numbers.

In 1857, Cayley introduced matrix notation, which allows for a harmonization and simplification of linear maps. Around the same time, Grassmann studied the barycentric calculus initiated by Mbius. He envisaged sets of abstract objects endowed with operations. In his work, the concepts of linear independence and dimension, as well as scalar products, are present. In fact, Grassmann's 1844 work exceeds the framework of vector spaces, since his consideration of multiplication led him to what are today called algebras. Peano was the first to give the modern definition of vector spaces and linear maps in 1888. An important development of vector spaces is due to the construction of function spaces by Lebesgue. This was later formalized by Banach and Hilbert, around 1920. At that time, algebra and the new field of functional analysis began to interact, notably with key concepts such as spaces of p-integrable functions and Hilbert spaces Vector spaces, including infinite-dimensional ones, then became a firmly established notion, and many mathematical branches started making use of this concept. In mathematics, a bivector is the vector part of a biquaternion. Now it is well known that the Lie algebra of the homogeneous Lorentz group can be considered to be that of bivectors under commutation [,]. William Rowan Hamilton coined both the terms vector and bivector. The first term was named with quaternions, and the second about a decade later, as in Lectures on Quaternions ([6]). In mathematics, fuzzy sets are sets whose elements have degrees of membership.

Fuzzy sets were introduced by Lotfi A. Zadeh[28] and Dieter Klaua[7] in 1965 as an extension of the classical notion of set. In classical set theory, the membership of elements in a set is assessed in binary terms according to a 
bivalent condition an element either belongs or does not belong to the set. By contrast, fuzzy set theory permits the gradual assessment of the membership of elements in a set; this is described with the aid of a membership function valued in the real unit interval $[0,1]$. Fuzzy sets generalize classical sets, since the indicator functions of classical sets are special cases of the membership functions of fuzzy sets, if the latter only take values 0 or 1 ([2]).

In fuzzy set theory, classical bivalent sets are usually called crisp sets. The fuzzy set theory can be used in a wide range of domains in which information is incomplete or imprecise, such as bioinformatics ([8]). In mathematics, a $t$-norm (also $T$-norm or, unabbreviated, triangular norm) is a kind of binary operation used in the framework of probabilistic metric spaces and in multi-valued logic, specifically in fuzzy logic. A $t$-norm generalizes intersection in a lattice and conjunction in logic. The name triangular norm refers to the fact that in the framework of probabilistic metric spaces $t$-norms are used to generalize triangle inequality of ordinary metric spaces. The author by using norms, investigated some properties of fuzzy submodules, fuzzy subrings, fuzzy ideals of subtraction semigroups, intuitionistic fuzzy subrings and ideals of a ring, fuzzy Lie algebra, fuzzy subgroups on direct product of groups, characterizations of intuitionistic fuzzy subsemirings of semirings and their homomorphisms, characterization of Q-fuzzy subrings (anti Q-fuzzy subrings) and fuzzy submodules of $R \times M([17,18,19,20,21,22,23,24,25,26]$ ).

In this work we introduce the concepts fuzzt sub-vector spaces, fuzzy sub-bivector spaces under $t$-norms and characterize some basic properties of them. The organization of this paper is as follows: In section 2, some preliminary definitions and concepts are given. In Section 3, we define the concepts fuzzy sub-vector spaces under $t$-norms. We prove that the intersection and sum of two fuzzy sub-vector spaces under $t$-norm are also fuzzy sub-vector space under $t$-norms. In this section we consider the relationship between subvector space and fuzzy sub-vector space under $t$-norm.

Also we obtain some results for fuzzy sub-vector spaces (under $t$-norms) under linear transformations between vector spaces. Finally in Section 4, we introduce fuzzy sub-bivector spaces under $t$-norms and investigate some basic properties of them and give new results on this subject.

\section{2 preliminary concepts}

In this section, we review some elementary aspects that are necessary for this paper. Throughout the paper we use $Z, R$ and $Q$ for set of integers numbers, reals numbers and rational numbers, respectively.

Definition 2.1. ([5]) A vector space or a linear space consists of the following:

(1) a field $\mathbb{F}$ of scalars.

(2) a set $V$ of objects called vectors.

(3) a rule (or operation) called vector addition; which associates with each pair of vectors $\alpha, \beta \in V ; \alpha+\beta \in V$, called the sum of $\alpha$ and $\beta$ in such a way that

(a) addition is commutative $\alpha+\beta=\beta+\alpha$.

(b) addition is associative $\alpha+(\beta+\gamma)=(\alpha+\beta)+\gamma$.

(c) there is a unique vector 0 in $V$, called the zero vector, such that $\alpha+0=$ for all $\alpha \in V$.

(d) for each vector $\alpha$ in $V$ there is a unique vector $(-\alpha)$ in $V$ such that $\alpha+(-\alpha)=0$.

(e) a rule (or operation), called scalar multiplication, which associates with each scalar $c$ in $\mathbb{F}$ and a vector $\alpha$ in $V$, a vector $c \bullet \alpha$ in $V$, called the product of $c$ and $\alpha$, in such a way that $1 \bullet \alpha=\alpha, \quad\left(c_{1} \bullet c_{2}\right) \bullet \alpha=c_{1} \bullet\left(c_{2} \bullet \alpha\right), c \bullet(\alpha+\beta)=$ $c \bullet \alpha+c \bullet \beta, \quad\left(c_{1}+c_{2}\right) \bullet \alpha=\left(c_{1} \bullet \alpha\right)+\left(c_{2} \bullet \alpha\right)$ for $\alpha, \beta \in V$ and $c, c_{1}, c_{2} \in F$. It is important to note as the definition states that a vector space is a composite object consisting of a field, a set of vectors and two operations with certain special properties. The same set of vectors may be part of a number of distinct vectors. We simply by default of notation just say $V$ a vector space over the field $\mathbb{F}$ and call elements of $V$ as vectors only as matter of convenience for the vectors in $V$.

Throughout this section, $\mathbb{F}$ is any field of characteristic zero.

Example 2.2. (a) Let $\mathbb{R}$ be the field of reals. $\mathbb{R}[x]$ the ring of polynomials. $\mathbb{R}[x]$ is a vector space over $\mathbb{R}$. Also $\mathbb{R}[x]$ is a vector space over the field of rationals $\mathbb{Q}$.

(b) Let $V=\mathbb{R} \times \mathbb{R} \times \mathbb{R}$. Then $V$ is a vector space over $\mathbb{R}$ or $\mathbb{Q}$ but $V$ is not a vector space over the complex field $\mathbb{C}$. (c) Let $M_{m \times n}=\left\{\left(a_{i j}\right) \mid a_{i j} \in \mathbb{Q}\right\}$ be the collection of all $m \times n$ matrices with entries from $\mathbb{Q}$. Then $M_{m \times n}$ is a vector space over $\mathbb{Q}$ but $M_{m \times n}$ is not a vector space over $\mathbb{R}$ or $\mathbb{C}$.

Definition 2.3. ([5]) Let $V$ be a vector space over the field $\mathbb{F}$. A subspace of $V$ is a subset $W$ of $V$ which is itself a vector space over $\mathbb{F}$ with the operations of vector addition and scalar multiplication on $V$. 
We have the following nice characterization theorem for subspaces.

Theorem 2.4. ([5]) Let $W$ be a non-empty subset of a vector $V$ over the field $\mathbb{F}$. Then $W$ is a subspace of $V$ if and only if for each pair $\alpha, \beta \in W$ and each scalar $c \in \mathbb{F}$ the vector $c \alpha+\beta \in W$.

Example 2.5. (a) Let $M_{n \times n}=\left\{\left(a_{i j}\right) \mid a_{i j} \in \mathbb{Q}\right\}$ be the vector space over $\mathbb{Q}$. Let $D_{n \times n}=\left\{\left(a_{i i}\right) \mid a_{i i} \in \mathbb{Q}\right\}$ be the set of all diagonal matrices with entries from $\mathbb{Q}$. Then $D_{n \times n}$ is a subspace of $M_{n \times n}$.

(b) Let $V=\mathbb{R}[x]$ be a polynomial ring, $\mathbb{R}[x]$ is a vector space over $\mathbb{Q}$. Then $W=\mathbb{Q}[x] \subset \mathbb{R}[x]$ is a subspace of $\mathbb{R}[x]$.

Definition 2.6. ([5]) Let $V$ and $W$ be two vector spaces over the field of $\mathbb{F}$. A map $f: V \rightarrow W$ is called a linear transformation if $f(c \alpha+\beta)=c f(\alpha)+f(\beta)$ for all $\alpha, \beta \in V$ and $c \in \mathbb{F}$.

Example 2.7. The map $f: \mathbb{R}^{3} \rightarrow \mathbb{R}^{3}$ defined by $f(x, y, z)=(x+y, y-z, x+2 y+z)$ is linear transformation of vector spaces over the field of $\mathbb{R}$.

Definition 2.8. ([11]) Let $X$ a non-empty sets. A fuzzy subset $\mu$ of $X$ is a function $\mu: X \rightarrow[0,1]$. Denote by $[0,1]^{X}$, the set of all fuzzy subset of $X$.

Definition 2.9. ([1]) A t-norm $T$ is a function $T:[0,1] \times[0,1] \rightarrow[0,1]$ having the following four properties:

(T1) $T(x, 1)=x$ (neutral element),

(T2) $T(x, y) \leq T(x, z)$ if $y \leq z$ (monotonicity),

(T3) $T(x, y)=T(y, x)$ (commutativity),

(T4) $T(x, T(y, z))=T(T(x, y), z)$ (associativity),

for all $x, y, z \in[0,1]$.

Recall that $T$ is idempotent if for all $x \in[0,1], T(x, x)=x$.

Example 2.10. For all $x, y \in[0,1]$ the basic $t$-norms are $T_{m}(x, y)=\min \{x, y\}$,

$T_{b}(x, y)=\max \{0, x+y-1\}$ and $T_{p}(x, y)=x y$, which are called standard intersection, bounded sum and algebraic product respectively.

Lemma 2.11. ([1]) Let $T$ be a t-norm. Then

$$
T(T(x, y), T(w, z))=T(T(x, w), T(y, z)),
$$

for all $x, y, w, z \in[0,1]$.

Definition 2.12. ([16]) The intersection of fuzzy subsets $\mu_{1}$ and $\mu_{1}$ in a set $X$ with respect to a $t$-norm $T$ we mean the fuzzy subset $\mu=\mu_{1} \cap \mu_{2}$ in the set $X$ such that for any $x \in X$

$$
\mu(x)=\left(\mu_{1} \cap \mu_{2}\right)(x)=T\left(\mu_{1}(x), \mu_{2}(x)\right) .
$$

Definition 2.13. ([11]) Let $f: V \rightarrow W$ be a linear transformation over the field $\mathbb{F}$. Let $\mu \in[0,1]^{V}$ and $\nu \in[0,1]^{W}$. Define $f(\mu) \in[0,1]^{W}$ and $f^{-1}(\nu) \in[0,1]^{V}$ as

$$
f(\mu)(w)=\left\{\begin{aligned}
\sup \{\mu(v) \mid v \in V, f(v)=w\} & \text { if } f^{-1}(w) \neq \emptyset \\
0 & \text { if } f^{-1}(w)=\emptyset
\end{aligned}\right.
$$

Also $f^{-1}(\nu)(v)=\nu(f(v))$.

Definition 2.14. ([27]) Let $\mu_{1}$ be a fuzzy subset of a set $X_{1}$ and $\mu_{2}$ be a fuzzy subset of a set $\mu_{2}$, then the fuzzy union of the fuzzy sets $\mu_{1}$ and $\mu_{2}$ is defined as a function.

$\mu_{1} \cup \mu_{2}: X_{1} \cup X_{2} \rightarrow[0,1]$ given by

$$
\left(\mu_{1} \cup \mu_{2}\right)(x)=\left\{\begin{aligned}
\max \left\{\mu_{1}(x), \mu_{2}(x)\right\} & \text { if } x \in X_{1} \cap X_{2} \\
\mu_{1}(x) & \text { if } x \in X_{1} \text { and } x \notin X_{2} \\
\mu_{2}(x) & \text { if } x \in X_{2} \text { and } x \notin X_{1} .
\end{aligned}\right.
$$

We illustrate this definition by the following example. 
Example 2.15. Let $X_{1}=\{1,2,3,4,5\}$ and $X_{2}=\{4,5,6,7,8\}$ be two sets. Define $\mu_{1}: X_{1} \rightarrow[0,1]$ by

$$
\mu_{1}(x)=\left\{\begin{aligned}
0.9 & \text { if } x=1,2 \\
0.8 & \text { if } x=3,4 \\
1 & \text { if } x=5
\end{aligned}\right.
$$

and

define $\mu_{2}: X_{2} \rightarrow[0,1]$ by

$$
\mu_{2}(x)=\left\{\begin{aligned}
1 & \text { if } x=4 \\
0.9 & \text { if } x=5,6 \\
0.8 & \text { if } x=7,8
\end{aligned}\right.
$$

It is easy to calculate $\mu_{1} \cup \mu_{1}$ and it is given as follows:

$$
\left(\mu_{1} \cup \mu_{1}\right)(x)=\left\{\begin{aligned}
1 & \text { if } x=4,5 \\
0.9 & \text { if } x=1,2,6 \\
0.8 & \text { if } x=3,7,8 .
\end{aligned}\right.
$$

Definition 2.16. ([27]) Let $V=V_{1} \cup V_{2}$ where $V_{1}$ and $V_{2}$ are two distinct set vector spaces defined over the same set $S$. That is $V_{1} \nsubseteq V_{2}$ and $V_{2} \nsubseteq V_{1}$ we may have $V_{1} \cap V_{2}=\emptyset$ or non empty. Then we call $V$ to be a set bivector space over $S$.

We illustrate this by the following example.

Example 2.17. Let $V=V_{1} \cup V_{2}$ where $V_{1}=\{(111),(000),(110),(11111),(00000),(11011),(11000),(100)\}$ and $V_{2}=\{(01),(10),(00),(1111),(0000),(0111),(111111),(000000),(1000),(0001)\}$ be set vector spaces over the set $S=$ $\{0,1\}$. Then $V=V_{1} \cup V_{2}$ is a set bivector space over the set $S$.

Now we proceed on to define the notion of set bivector bisubspaces of a set bivector space.

Definition 2.18. ([27]) Let $V=V_{1} \cup V_{2}$ be a set bivector space defined over the set $S$. A proper biset $W=$ $W_{1} \cup W_{2}\left(W_{1} \subset V_{1}\right.$ and $\left.W_{2} \subset V_{2}\right)$ such that $W_{1}$ and $W_{2}$ are distinct and contained in $V$ is said to be a set bivector bisubspace of $V$ (or set bivector subspace) if $W$ is a set bivector space defined over $S$.

\section{3 fuzzy sub-vector spaces under $t$-norms}

In this section we introduce definition and properties of fuzzy vector spaces under $t$-norms. The study of fuzzy vector spaces started as early as 1977. For more about fuzzy sub-vector spaces refer [9, 10, 13, 14, 15].

Definition 3.1. The fuzzy subset $K: \mathbb{F} \rightarrow[0,1]$ is called fuzzy subfield of $\mathbb{F}$ under $t$-norm $T$ if (1) $K(1)=1$,

(2) $K(c-d) \geq T(K(c), K(d))$ for all $c, d \in \mathbb{F}$ and

(3) $K\left(c d^{-1}\right) \geq T(K(c), K(d))$ for all $c, d \in \mathbb{F}$ where $d \neq 0$.

Example 3.2. Let $V=\mathbb{R}$ be vector space over field $\mathbb{R}$. Define $K: V \rightarrow[0,1]$ by

$$
K(x)=\left\{\begin{aligned}
1 & \text { if } x \in \mathbb{R}^{\geq 0} \\
0.7 & \text { if } x \in \mathbb{R}^{<0} .
\end{aligned}\right.
$$

Let $T(a, b)=T_{p}(a, b)=a b$ for all $a, b \in[0,1]$. Then $K$ is fuzzy subfield of $\mathbb{R}$ under $t$-norm $T$.

Definition 3.3. Let $K: \mathbb{F} \rightarrow[0,1]$ be a fuzzy subset of $\mathbb{F}$. The fuzzy subset $A: V \rightarrow[0,1]$ is called fuzzy sub-vector space over a fuzzy subfield $K$ of $\mathbb{F}$ under $t$-norm $T$ if

(1) $A(0)>0$,

(2) $A(x-y) \geq T(A(x), A(y))$,

(3) $A(c x) \geq T(K(c), A(x))$ for all $x, y \in V$ and $c \in \mathbb{F}$.

Denote by FST $(V)$, the set of all fuzzy sub-vector space of $V$ with respect to a $t$-norm $T$. 
Example 3.4. Let $V=\mathbb{R} \times \mathbb{R}$ be a vectorspace over $\mathbb{R}$. Define $\mu: \mathbb{R} \times \mathbb{R} \rightarrow[0,1]$ as

$$
\mu(x)=\left\{\begin{array}{rl}
1 & x \in\{(a, 0) \mid a \in \mathbb{R}\} \\
0.3 & \text { otherwise. }
\end{array}\right.
$$

Let $T(a, b)=T_{p}(a, b)=a b$ for all $a, b \in[0,1]$. Then $\mu \in F S T(V)$.

Let $\mu \in F S T(V)$. Then $B=\{x \mid x \in V, \mu(x)=1\}$ is a subspace of $V$.

Proof 3.5. Let $x, y \in B$ and $c \in \mathbb{F}$. Then $\mu(c x+y) \geq T(\mu(c x), \mu(y))=T(\mu(c x), 1)=1$. Therefore $\mu(c x+y)=1$ and from Theorem 2.4 we get that $B$ is a subspace of $V$.

Let $\mu \in F S T(V)$ and $T$ be idempotent. If for all $c \in \mathbb{F}$ we have $K(c) \geq t$, then for any $t \in[0,1], \mu^{t}=\{x \mid v \in$ $V, \mu(x) \geq t\}$ will be a subspace of the $V$.

Proof 3.6. Let $\alpha, \beta \in \mu^{t}$ and $c \in \mathbb{F}$. Then $\mu(\alpha), \mu(\beta) \geq t$. Now

$$
\begin{gathered}
\mu(c \alpha+\beta) \geq T(\mu(c \alpha), \mu(\beta)) \geq T(T(K(c), \mu(\alpha)), t) \\
=T(K(c), T(\mu(\alpha), t)) \geq T(K(c), T(t, t))=T(K(c), t) \geq T(t, t)=t .
\end{gathered}
$$

Thus $c \alpha+\beta \in \mu^{t}$ and Theorem 2.4 gives that $\mu^{t}$ is a subspace of the $V$.

Theorem 3.7. Let $V$ be a subspace over field $\mathbb{F}$ and $W$ be a subset of $V$. If $\mu: W \rightarrow\{0,1\}$ be the characteristic function and $K(c)=1$ for all $c \in \mathbb{F}$, then $\mu \in F S T(V)$ if and only if $W$ is a subspace of $V$.

Proof 3.8. Let $\mu \in F S T(V)$ and we prove that $W$ is a subspace of $V$. Let $\alpha, \beta \in W$ and $c \in \mathbb{F}$. Then $\mu(\alpha)=\mu(\beta)=1$ and

$$
\mu(c \alpha+\beta) \geq T(\mu(c \alpha), \mu(\beta)) \geq T(T(K(c), \mu(\alpha)), \mu(\beta))=T(T(1,1), 1)=T(1,1)=1
$$

so $\mu(c \alpha+\beta)=1$ and from Theorem 2.4 we get $W$ is a subspace of $V$.

Conversely, let $W$ is a subspace of $V$ and we prove that $\mu \in F S T(V)$. Since $0 \in W$ so $\mu(0)=1>0$. Let $\alpha, \beta \in V$ and we investigate the following conditions:

(1) If $\alpha, \beta \in W$, then $\alpha-\beta \in W$ and we have $\mu(\alpha-\beta)=1 \geq 1=T(1,1)=T(\mu(\alpha), \mu(\beta))$.

(2) If $\alpha \notin W$ and $\beta \in W$, then $\alpha-\beta \notin W$ and then $\mu(\alpha-\beta)=0 \geq 0=T(0,1)=T(\mu(\alpha), \mu(\beta))$.

(3) Finally, if $\alpha, \beta, \notin W$, then $\mu(\alpha-\beta) \geq 0=T(0,0)=T(\mu(\alpha), \mu(\beta))$.

Thus from (1)-(3) we have that $\mu(\alpha-\beta) \geq T(\mu(\alpha), \mu(\beta))$. Now let $\alpha \in V$ and $c \in \mathbb{F}$.

(1) If $\alpha \in W$, then $c \alpha \in W$ and so $\mu(c \alpha)=1 \geq 1=T(1,1)=T(K(c), \mu(\alpha))$.

(2) If $\alpha \notin W$, then $c \alpha \notin W$ so $\mu(c \alpha)=0 \geq 0=T(1,0)=T(K(c), \mu(\alpha))$.

Then from (1) and (2) we obtain $\mu(c \alpha) \geq T(K(c), \mu(\alpha))$. Therefore $\mu \in F S T(V)$.

Definition 3.9. Let $W$ be a subspace of $V$ over a field $\mathbb{F}$ and $A: V \rightarrow[0,1]$ be a fuzzy sub-vector space over a fuzzy subfield $K$ of $\mathbb{F}$. Define $\hat{A}: V / W \rightarrow[0,1]$ as

$$
\hat{A}(v+W)=\left\{\begin{aligned}
1 & \text { if } v=w \\
T(A(v), A(w)) & \text { if } v \neq w
\end{aligned}\right.
$$

and is called the fuzzy quotient vector space.

Let $A \in F S T(V)$ and $T$ be idempotent. Then $\hat{A} \in F S T(V / W)$.

Proof 3.10. Let $v_{1}, v_{2} \in V$ and $w \in W$. If $v_{1}=w$, then $\hat{A}(0)=1>0$. If $v_{1} \neq w \neq v_{2}$, then

$$
\begin{gathered}
\left.\hat{A}\left(\left(v_{1}+W\right)-\left(v_{2}+W\right)\right)=\hat{A}\left(\left(v_{1}-v_{2}\right)+W\right)\right)=T\left(A\left(v_{1}-v_{2}\right), A(w)\right) \\
\geq T\left(T\left(A\left(v_{1}\right), A\left(v_{2}\right)\right), T(A(w), A(w))\right)=T\left(T\left(A\left(v_{1}\right), A(w)\right), T\left(A\left(v_{2}\right), A(w)\right)\right) \\
=T\left(\hat{A}\left(v_{1}+W\right), \hat{A}\left(v_{2}+W\right)\right) .
\end{gathered}
$$

Also if $c \in \mathbb{F}$, then

$$
\begin{gathered}
\hat{A}\left(c\left(v_{1}+W\right)\right)=\hat{A}\left(c v_{1}+W\right)=T\left(A\left(c v_{1}\right), A(w)\right) \\
\geq T\left(T\left(K(c), A\left(v_{1}\right)\right), A(w)\right)=T\left(K(c), \hat{A}\left(v_{1}+W\right)\right) .
\end{gathered}
$$

Thus $\hat{A} \in F S T(V / W)$. 
Let $\mu_{1}, \mu_{2} \in F S T(V)$ and $T$ be idempotent. Then $\left(\mu_{1} \cap \mu_{2}\right) \in F S T(V)$.

Proof 3.11. Let $x, y \in V$ and $c \in \mathbb{F}$.

(1) $\left(\mu_{1} \cap \mu_{2}\right)(0)=T\left(\mu_{1}(0), \mu_{2}(0)\right)>0\left(\right.$ since $\left.\mu_{1}(0), \mu_{2}(0)>0\right)$.

(2)

$$
\begin{gathered}
\left(\mu_{1} \cap \mu_{2}\right)(x-y)=T\left(\mu_{1}(x-y), \mu_{2}(x-y)\right) \geq T\left(T\left(\mu_{1}(x), \mu_{1}(y)\right), T\left(\mu_{2}(x), \mu_{2}(y)\right)\right) \\
=T\left(T\left(\mu_{1}(x), \mu_{2}(x)\right), T\left(\mu_{1}(y), \mu_{2}(y)\right)\right) \quad(\text { from Lemma 2.11) } \\
=T\left(\left(\mu_{1} \cap \mu_{2}\right)(x),\left(\mu_{1} \cap \mu_{2}\right)(y)\right)
\end{gathered}
$$

(3)

$$
\begin{gathered}
\left(\mu_{1} \cap \mu_{2}\right)(c x)=T\left(\mu_{1}(c x), \mu_{2}(c x)\right) \geq T\left(T\left(K(c), \mu_{1}(x)\right), T\left(K(c), \mu_{2}(x)\right)\right)= \\
T\left(T(K(c), K(c)), T\left(\mu_{1}(x), \mu_{2}(x)\right)\right)=T\left(K(c),\left(\mu_{1} \cap \mu_{2}\right)(x)\right) .
\end{gathered}
$$

Hence $\left(\mu_{1} \cap \mu_{2}\right) \in F S T(V)$.

Corollary 3.12. Let $\left\{\mu_{i} \mid i \in I_{n}=1,2, \ldots, n\right\} \subseteq F S T(V)$. Then $\left(\cap_{i \in I_{n}} \mu_{i}\right) \in F S T(V)$.

Definition 3.13. Let $\mu_{1}, \mu_{2} \in F S T(V)$. The sum of $\mu_{1}$ and $\mu_{2}$ is defined as follows:

$$
\left(\mu_{1}+\mu_{2}\right)(x)=\sup \left\{T\left(\mu_{1}(y), \mu_{2}(z)\right) \mid x=y+z \in V\right\} .
$$

Let $\mu_{1}, \mu_{2} \in F S T(V)$ and $T$ be idempotent. Then $\left(\mu_{1}+\mu_{2}\right) \in F S T(V)$.

Proof 3.14. (1) Since $\mu_{1}(0), \mu_{2}(0)>0$, Then

$$
\left(\mu_{1}+\mu_{2}\right)(0)=\sup \left\{T\left(\mu_{1}(0), \mu_{2}(0)\right) \mid 0=0+0\right\}>0 .
$$

(2) Let $x_{1}, x_{2}, y_{1}, y_{2}, z_{1}, z_{2} \in V$. Then

$$
\begin{gathered}
\left(\mu_{1}+\mu_{2}\right)\left(x_{1}-x_{2}\right) \\
=\sup \left\{T\left(\mu_{1}\left(y_{1}-y_{2}\right), \mu_{2}\left(z_{1}-z_{2}\right)\right) \mid x_{1}-x_{2}=y_{1}-y_{2}+z_{1}-z_{2}\right\} \\
\geq \sup \left\{T\left(T\left(\mu_{1}\left(y_{1}\right), \mu_{1}\left(y_{2}\right)\right), T\left(\mu_{2}\left(z_{1}\right), \mu_{2}\left(z_{2}\right)\right)\right) \mid x_{1}-x_{2}=y_{1}+z_{1}-\left(y_{2}+z_{2}\right)\right\} \\
(\text { from Lemma } 2.11) \\
=\sup \left\{T\left(T\left(\mu_{1}\left(y_{1}\right), \mu_{2}\left(z_{1}\right)\right), T\left(\mu_{1}\left(y_{2}\right), \mu_{2}\left(z_{2}\right)\right)\right) \mid x_{1}-x_{2}=y_{1}+z_{1}-\left(y_{2}+z_{2}\right)\right\} \\
\left.=T\left(\sup \left\{T\left(\mu_{1}\left(y_{1}\right), \mu_{2}\left(z_{1}\right)\right) \mid x_{1}=y_{1}+z_{1}\right)\right\}, \sup \left\{T\left(\mu_{1}\left(y_{2}\right), \mu_{2}\left(z_{2}\right)\right) \mid x_{2}=y_{2}+z_{2}\right\}\right) \\
=T\left(\left(\mu_{1}+\mu_{2}\right)\left(x_{1}\right),\left(\mu_{1}+\mu_{2}\right)\left(x_{2}\right)\right) .
\end{gathered}
$$

(3) Let $x, y, z \in V$ and $c \in \mathbb{F}$.

$$
\begin{gathered}
\left(\mu_{1}+\mu_{2}\right)(c x)=\sup \left\{T\left(\mu_{1}(c y), \mu_{2}(c z)\right) \mid c x=c y+c z\right\} \\
\geq \sup \left\{T\left(T\left(K(c), \mu_{1}(y)\right), T\left(K(c), \mu_{2}(z)\right)\right) \mid x=y+z\right\} \\
\quad(\text { from Lemma 2.11) } \\
=\sup \left\{T\left(T(K(c), K(c)), T\left(\mu_{1}(y), \mu_{2}(z)\right)\right) \mid x=y+z\right\} \\
\quad(\text { since } \quad T \quad \text { is idempotent }) \\
=\sup \left\{T\left(K(c), T\left(\mu_{1}(y), \mu_{2}(z)\right)\right) \mid x=y+z\right\} \\
=T\left(K(c),\left(\mu_{1}+\mu_{2}\right)(x)\right) .
\end{gathered}
$$

Then $\left(\mu_{1}+\mu_{2}\right) \in F S T(V)$. 
Let $f$ be an epimorphism linear transformation from vector space $V$ into vector space $W$ over field $\mathbb{F}$. If $\mu \in$ $F S T(V)$, then $f(\mu) \in F S T(W)$.

Proof 3.15. (1) $f(\mu)(0)=\sup \{\mu(0) \mid 0 \in V, f(0)=0\}>0$.

(2) Let $w_{1}, w_{2} \in W$.

$$
\begin{gathered}
f(\mu)\left(w_{1}-w_{2}\right)=\sup \left\{\mu\left(v_{1}-v_{2}\right) \mid v_{1}, v_{2} \in V, f\left(v_{1}\right)=w_{1}, f\left(v_{2}\right)=w_{2}\right\} \\
\geq \sup \left\{T\left(\mu\left(v_{1}\right), \mu\left(v_{2}\right)\right) \mid v_{1}, v_{2} \in V, f\left(v_{1}\right)=w_{1}, f\left(v_{2}\right)=w_{2}\right\} \\
=T\left(\sup \left\{\mu\left(v_{1}\right) \mid f\left(v_{1}\right)=w_{1}\right\}, \sup \left\{\mu\left(v_{2}\right) \mid f\left(v_{2}\right)=w_{2}\right\}\right) \\
=T\left(f(\mu)\left(w_{1}\right), f(\mu)\left(w_{2}\right)\right) .
\end{gathered}
$$

(3) Let $v \in V$ and $c \in \mathbb{F}$.

$$
\begin{gathered}
f(\mu)(c w)=\sup \{\mu(c v)) \mid c v \in V, f(c v)=c w\} \\
\geq \sup \{T(K(c), \mu(v)) \mid v \in V, f(v)=w\} \\
=T(K(c), \sup \{\mu(v) \mid v \in V, f(v)=w\}) \\
=T(K(c), f(\mu)(w)) .
\end{gathered}
$$

Therefore $f(\mu) \in F S T(W)$.

Let $f$ be a linear transformation from vector space $V$ into vector space $W$ over field $\mathbb{F}$. If $\nu \in F S T(W)$, then $f^{-1}(\nu) \in F S T(V)$.

Proof 3.16. (1) $f^{-1}(\nu)(0)=\nu(f(0))=\nu(0)>0$.

Let $v_{1}, v_{2} \in V$. Then

(2)

$$
\begin{aligned}
& f^{-1}(\nu)\left(v_{1}-v_{2}\right)=\nu\left(f\left(v_{1}-v_{2}\right)\right)=\nu\left(f\left(v_{1}\right)-f\left(v_{2}\right)\right) \\
& \geq T\left(\nu \left(f\left(v_{1}\right), \nu\left(f\left(v_{2}\right)\right)=T\left(f^{-1}(\nu)\left(v_{1}\right), f^{-1}(\nu)\left(v_{2}\right)\right) .\right.\right.
\end{aligned}
$$

(3) Let $v \in V$ and $c \in \mathbb{F}$. Then

$$
f^{-1}(\nu)(c v)=\nu(f(c v))=\nu(c f(v))=T\left(K(c), \nu(f(v))=T\left(K(c), f^{-1}(\nu)(v)\right) .\right.
$$

Thus $f^{-1}(\nu) \in F S T(V)$.

\section{Fuzzy sub-bivector spaces under $t$-norms}

In this section we define fuzzy sub-bivector spaces under $t$-norms.

Definition 4.1. Let $V=V_{1} \cup V_{2}$ be a set bivector space over field $\mathbb{F}$ and $\mu: V \rightarrow[0,1]$ fuzzy subset of $V$. Then we say that $\mu \in F S T(V)$ if there exists two fuzzy subsets $\mu_{1}$ (of $V_{1}$ ) and $\mu_{2}$ (of $V_{2}$ ) such that

(1) $\mu_{1} \in F S T\left(V_{1}\right)$

(2) $\mu_{2} \in F S T\left(V_{2}\right)$ and

(3) $\mu=\mu_{1} \cup \mu_{2}$. 
Example 4.2. Let $V=V_{1} \cup V_{2}$ where $V_{1}=\mathbb{R}[x]$ and $V_{2}=\mathbb{R} \times \mathbb{R}$ be two vector spaces over field $\mathbb{R}$. Define $\mu_{1}: \mathbb{R}[x] \rightarrow[0,1]$ by

$$
\mu_{1}(p(x))=\left\{\begin{aligned}
\frac{1}{\operatorname{deg}(p(x))} & \text { if } \operatorname{deg}(p(x)) \neq 0 \\
0.8 & \text { if } \operatorname{deg}(p(x))=0
\end{aligned}\right.
$$

Also define $\mu_{2}: \mathbb{R} \times \mathbb{R} \rightarrow[0,1]$ as

$$
\mu_{2}(x)= \begin{cases}0.9 & x \in\{(0, a) \mid a \in \mathbb{R}\} \\ 0.6 & \text { otherwise }\end{cases}
$$

Let $T(a, b)=T_{p}(a, b)=a b$ for all $a, b \in[0,1]$. That is, there exist $\mu_{1} \in F S T\left(V_{1}\right)$ and $\mu_{2} \in F S T\left(V_{2}\right)$ such that $\mu=\mu_{1} \cup \mu_{2}$.

Remark 4.3. The Proposition 3.5 is not true for fuzzy sub-bivector spaces. For example take $V=V_{1} \cup V_{2}$ where $V_{1}=\mathbb{R}[x]$ and $V_{2}=\mathbb{R}[y]$ be two vector spaces over field $\mathbb{R}$. Define $\mu=\mu_{1} \cup \mu_{2}: V \rightarrow[0,1]$ by

$$
\mu(p)= \begin{cases}0.25 & \text { if } p \in V_{1} \\ 0.12 & \text { if } p \in V_{2}\end{cases}
$$

If $T(a, b)=T_{p}(a, b)=a b$ for all $a, b \in[0,1]$, then $\mu \in F S T(V)$. Now for $t=0.16 \in[0,1]$ we have that $\mu^{t}=\{p \in V \mid \mu(p) \geq 0.16\}=V_{1}=\mathbb{R}[x]$ such that is not a sub-bispace of the bispace $V=V_{1} \cup V_{2}$.

Definition 4.4. A fuzzy subset $\mu$ of a vector space $V$ is said to be a fuzzy sub-bivector space of the vector space $V$ if there exists two fuzzy subvector spaces $\mu_{1}$ and $\mu_{2}$ of $\mu\left(\mu_{1} \neq \mu\right)$ and $\left.\mu_{2} \neq \mu\right)$ such that $\mu=\mu_{1} \cup \mu_{2}$. Here by the term fuzzy sub-vector space $\lambda$ of $\mu$ we mean that $\lambda$ is a fuzzy subvector space $V$ and $(\lambda \subseteq \mu)$ is also a fuzzy sub-vector space $V$.

Let $\mu=\mu_{1} \cup \mu_{2}$ be a fuzzy sub-bispace of a vector space $V$ undet $t$-norm $T$, where $\mu_{1}$ and $\mu_{1}$ are fuzzy subspace of a vector space $V$ undet $t$-norm $T$. For $t \in\left[0, \min \left\{\mu_{1}(0), \mu_{2}(0)\right\}\right]$, the level subset $\mu^{t}$ of $\mu$ can be represented as the union of two subspaces of the vector space $V$. That is $\mu^{t}=\mu_{1}^{t} \cup \mu_{2}^{t}$.

Proof 4.5. Let $\mu \in F S T(V)$ and $t \in\left[0, \min \left\{\mu_{1}(0), \mu_{2}(0)\right\}\right]$. Then there exists $\mu_{1} \in F S T\left(V_{1}\right)$ and $\mu_{2} \in F S T\left(V_{2}\right)$ such that $\mu=\mu_{1} \cup \mu_{2}$. Then $x \in \mu^{t}$ if and only if $\mu(x) \geq t$ if and only if $\max \left\{\mu_{1}(x), \mu_{2}(x)\right\} \geq t$ if and only if $\mu_{1}(x) \geq t$ or $\mu_{2}(x) \geq t$ if and only if $x \in \mu_{1}^{t}$ or $x \in \mu_{2}^{t}$ if and only if $x \in \mu_{1}^{t} \cup \mu_{2}^{t}$. Therefore $\mu^{t}=\mu_{1}^{t} \cup \mu_{2}^{t}$.

Example 4.6. Let $V=\mathbb{R}$ be vector space over field $\mathbb{R}$. Define $\mu: \mathbb{R} \rightarrow[0,1]$ by

$$
\mu(x)=\left\{\begin{aligned}
1 & \text { if } x \in \mathbb{R}^{\geq 0} \\
0.5 & \text { if } x \in \mathbb{R}^{<0}
\end{aligned}\right.
$$

It can be verified that $\mu$ is a fuzzy sub-bispace of the $V$ (under $T(a, b)=T_{p}(a, b)=a b$ for all $\left.a, b \in[0,1]\right)$, as there exists two fuzzy subspaces $\mu_{1}$ and $\mu_{1}$ of $\mu\left(\mu_{1} \neq \mu\right.$ and $\left.\mu_{2} \neq \mu\right)$ such that $\mu=\mu_{1} \cup \mu_{2}$ where $\mu_{1}$ and $\mu_{1}$ are as given below.

$$
\mu_{1}(x)=\left\{\begin{aligned}
1 & \text { if } x \in \mathbb{R}^{\geq 0} \\
0.25 & \text { if } x \in \mathbb{R}^{<0}
\end{aligned}\right.
$$

and

$$
\mu_{2}(x)=\left\{\begin{aligned}
0.75 & \text { if } x \in \mathbb{R}^{\geq 0} \\
0.5 & \text { if } x \in \mathbb{R}^{<0} .
\end{aligned}\right.
$$

For $t=0.5$ we have $\mu^{t}=\mathbb{R}, \mu_{1}^{t}=\mathbb{R}^{\geq 0}$ and $\mu_{2}^{t}=\mathbb{R}$ and then $\mu^{t}=\mu_{1}^{t} \cup \mu_{2}^{t}$.

Now we give the condition for two fuzzy sub-vector spaces $\mu_{1}$ and $\mu_{2}$ of a fuzzy sub-vector spaces $\mu\left(\mu_{1} \neq \mu\right.$ and $\left.\mu_{2} \neq \mu\right)$ to be such that $\mu=\mu_{1} \cup \mu_{2}$.

Let $\mu \in \operatorname{SFT}(V)$ with $3 \leq O\left(\operatorname{Im}(\mu)<\infty\right.$. Then there exists two fuzzy subvector spaces $\mu_{1}$ and $\mu_{2}$ (under $t$-norm $T)$ of a fuzzy subvector spaces $\mu\left(\mu_{1} \neq \mu\right.$ and $\left.\mu_{2} \neq \mu\right)$ to be such that $\mu=\mu_{1} \cup \mu_{2}$. 
Proof 4.7. Let $\mu \in S F T(V)$ with $3 \leq O\left(\operatorname{Im}(\mu)<\infty\right.$. Suppose $\operatorname{Im}(\mu)=\left\{a_{1}, a_{2}, \ldots, a_{n}\right\}$ where $3 \leq n<\infty$ and $a_{1}>a_{2}>\ldots>a_{n}$. Choose $b_{1}, b_{2}, \ldots, b_{n} \in[0,1]$ be such that $a_{1}>b_{1}>a_{2}>b_{2}>a_{3}>b_{3}>\ldots>a_{n}$. Now define $\mu_{1}, \mu_{2}: V \rightarrow[0,1]$ by

and

$$
\mu_{1}(x)=\left\{\begin{aligned}
a_{1} & \text { if } x \in \mu^{a_{1}} \\
b_{2} & \text { if } x \in \mu^{a_{2}} \text { and } x \notin \mu^{a_{1}} \\
\mu(x) & \text { otherwise }
\end{aligned}\right.
$$

and

$$
\mu_{2}(x)=\left\{\begin{aligned}
b_{1} & \text { if } x \in \mu^{a_{1}} \\
a_{2} & \text { if } x \in \mu^{a_{2}} \text { and } x \notin \mu^{a_{1}} \\
\mu(x) & \text { otherwise. }
\end{aligned}\right.
$$

Then we obtain $\mu_{1}$ and $\mu_{2}$ such that $\mu_{1}, \mu_{2} \subset \mu$ and $\mu=\mu_{1} \cup \mu_{2}$.

Theorem 4.8. Let $V$ be vector space over field $\mathbb{F}$ and $\mu: V \rightarrow[0,1]$ be fuzzy subset of $V$ with $3 \leq$ $O(\operatorname{Im}(\mu)<\infty$. Then $\mu \in F S T(V)$ ( $V$ as vector space) if and only if $\mu \in F S T(V)$ ( $V$ as bivector space).

Proof 4.9. Let $\mu \in F S T(V)$ ( $V$ as vector space) then exists two fuzzy subvector spaces $\mu_{1}$ and $\mu_{2}$ (under t-norm $T)$ of $\mu\left(\mu_{1} \neq \mu\right.$ and $\left.\mu_{2} \neq \mu\right)$ such that $\mu=\mu_{1} \cup \mu_{2}$. Therefore $\mu \in F S T(V)$ (V as bivector space). Conversely, let $\mu \in F S T(V)$ ( $V$ as bivector space). Since every fuzzy sub-bispace of a $V$ is a fuzzy subspace of $V$ so $\mu \in F S T(V)$ ( $V$ as vector space)

We shall illustrate this theorem by example.

Example 4.10. Let $V=\mathbb{R} \times \mathbb{R} \times \mathbb{R}$ be vector space over field $\mathbb{R}$. Define $\mu: V \rightarrow[0,1]$ by

$$
\mu(x)= \begin{cases}0.9 & \text { if } x \in\left\{(a, a, 0) \mid a \in \mathbb{R}^{\geq 0}\right\} \\ 0.8 & \text { if } x \in\left\{(a, a, 0) \mid a \in \mathbb{R}^{<0}\right\} \\ 0.6 & \text { otherwise. }\end{cases}
$$

Also define $\mu_{1}, \mu_{2}: V \rightarrow[0,1]$ by

$$
\mu_{1}(x)= \begin{cases}0.9 & \text { if } x \in\left\{(a, a, 0) \mid a \in \mathbb{R}^{\geq 0}\right\} \\ 0.6 & \text { otherwise }\end{cases}
$$

and

$$
\mu_{2}(x)=\left\{\begin{aligned}
0.8 & \text { if } x \in\left\{(a, a, 0) \mid a \in \mathbb{R}^{<0}\right\} \\
0.45 & \text { otherwise. }
\end{aligned}\right.
$$

Then $\mu, \mu_{1}, \mu_{2} \in F S T(V)$ (under $T(a, b)=T_{p}(a, b)=$ ab for all $a, b \in[0,1]$ ). It is easy to prove that $\mu_{1}, \mu_{2} \subset \mu$ and $\mu=\mu_{1} \cup \mu_{2}$.

\section{Conclusion}

In this study, we define sub-vector spaces and sub-bivector spaces uner $t$-norms. we show the relainship between sub-vector spaces uner $t$-norms and sub-vector spaces. Next we prove that the intersection and sum of two sub-vector spaces uner $t$-norms is also sub-vector spaces uner $t$-norms. Later we investigate ub-vector spaces uner $t$-norms under homomorphisms of vector spaces. Finally, we introduce sub-bivector spaces uner $t$-norms and obtain some results about them. 


\section{Open Problem}

S. Melliani, A. El Allaoui and L. S. Chadli investigated fuzzy semigroups and fuzzy dynamical systems, fuzzy $\alpha$-semigroups of operators $([30,40,50])$. Now the open problem is that one can investigate norms over fuzzy semigroups and fuzzy dynamical systems, fuzzy $\alpha$-semigroups of operators as the author investigated norms over sub-vector spaces and sub-bivector spaces.

Acknowledgment. We would like to thank the reviewers for carefully reading the manuscript and making several helpful comments to increase the quality of the paper.

\section{References}

[1] M. T. Abu Osman, On some products of fuzzy subgroups, Fuzzy Sets and Systems, 24 (1987), 79-86. https://doi.org/10.1016/0165-0114(87)90115-1

[2] D. Dubois and H. Prade, Fuzzy Sets and Systems, Academic Press, New York, 1988.

[3] A. El Allaoui, S. Melliani and L. S. Chadli, Fuzzy dynamical systems and Invariant attractor sets for fuzzy strongly continuous semigroups, Journal of Fuzzy Set Valued Analysis, 2(2016), 148-155. https://doi.org/10.5899/2016/jfsva-00294

[4] A. El Allaoui, S. Melliani and L. S. Chadli, Fuzzy a-semigroups of operators, General Letters in Mathematics, 2(2017), 42-49. https://doi.org/10.31559/glm2016.2.2.2

[5] K. Enneth Hoffman and R. Kunze, Linear Algebra, Prentice-Hall, Inc. , Englewood Cliffs, New Jersey, 1961.

[6] W. R. Hamilton, On the geometrical interpretation of some results obtained by calculation with biquaternions, Proceedings of the Royal Irish Academy, 5(1853), 388-390.

[7] D. Klaua, An early approach toward graded identity and graded membership in set theory, Fuzzy Sets and Systems, 161(2010), 2369-2379. https://doi.org/10.1016/j.fss.2009.12.005

[8] R. Liang, S. Lu, X. Wang, Y. Lu, V. Mandal, D. Patacsil, and D. Kumar, A Fuzzy-Set-Theory-Based Approach to Differential Gene Expression Data Analysis, BMC Bioinformatics, 7 (Suppl 4): S7. 2006. https://doi.org/10.1186/1471-2105-7-s4-s7

[9] D. S. Malik and J. N. Mordeson, Fuzzy subfields, Fuzzy Sets and Systems, 37(1990), 383-388. https://doi.org/10.1016/0165-0114(90)90034-4

[10] D. S. Malik and J. N. Mordeson, Fuzzy Maximal, Radical and Primary Ideals of a Ring, Inform. Sci., 53(1991), 237-250. https://doi.org/10.1016/0020-0255(91)90038-v

[11] D. S. Malik and J. N. Mordeson, Fuzzy Commutative Algebra, World Science publishing Co.Pte.Ltd.,(1995). https://doi.org/10.1142/9789812815897

[12] S. Melliani, A. El Allaoui and L. S. Chadli, Relation Between Fuzzy Semigroups and Fuzzy Dynamical Systems, Nonlinear Dynamics and Systems Theory, 17(2017), 60-69.

[13] J. N. Mordeson, Fuzzy transcendental field extensions, Chinese J. Math., 20(1992), 55-68.

[14] J. N. Mordeson, Bases of Fuzzy Vector Spaces, Inform. Sci., 67(1993), 87-92. https://doi.org/10.1016/0020-0255(93)90085-z 
[15] S. Nanda, Fuzzy algebras over fuzzy fields, Fuzzy Sets and Systems, 37(1990), 99-103. https://doi.org/10.1016/0165-0114(90)90067-g

[16] S. G. Pushkov, Fuzzy modules with respect to a t-norm and some of their properties, Journal of Mathematical Sciences, 154(2008), 374-378. https://doi.org/10.1007/s10958-008-9175-3

[17] R. Rasuli, Fuzzy Ideals of Subtraction Semigroups with Respect to a t-norm and A t-conorm, The Journal of Fuzzy Mathematics Los Angeles, 24 (4) (2016), 881-892.

[18] R. Rasuli, Fuzzy modules over a t-norm, Int. J. Open Problems Compt. Math., 9 (3) (2016), 12-18. https://doi.org/10.12816/0033740

[19] R. Rasuli, Fuzzy Subrings over a t-norm, The Journal of Fuzzy Mathematics Los Angeles, 24 (4) (2016), 995-1000.

[20] R. Rasuli, Norms over intuitionistic fuzzy subrings and ideals of a ring, Notes on Intuitionistic Fuzzy Sets, 22 (5) (2016), 72-83.

[21] R. Rasuli, Norms over fuzzy Lie algebra, Journal of New Theory, 15(2017), 32-38.

[22] R. Rasuli, Fuzzy subgroups on direct product of groups over a t-norm, Journal of Fuzzy Set Valued Analysis, 3(2017), 96-101. https://doi.org/10.5899/2017/jfsva-00339

[23] R. Rasuli, Characterizations of intuitionistic fuzzy subsemirings of semirings and their homomorphisms by norms, Journal of New Theory, 18(2017), 39-52.

[24] R. Rasuli, intuitionistic fuzzy subrings and ideals of a ring under norms, LAP LAMBERT Academic publishing, 2017, ISBN: 978-620-2-06926-7.

[25] R. Rasuli, Characterization of Q-Fuzzy subrings (Anti Q-Fuzzy Subrings) with respect to a T-norm (T-Conorms), Journal of Information and Optimization Science, 31(2018), 1-11. https://doi.org/10.1080/02522667.2016.1228316

[26] R. Rasuli, T-Fuzzy Submodules of $R \times M$, Journal of New Theory, 22(2018), 92-102.

[27] W. B. Vasantha Kandasamy, Smarandache bialgebraic structures, American Research Press, Rehoboth, NM, 2003.

[28] L. A. Zadeh, Fuzzy sets, Inform. Control., 8(1965), 338-353. 